\title{
Impact of Definitive Drug-Drug Interaction Testing on Medication Management and Patient Care
}

\author{
Renée J. G. Arnold ${ }^{1,2} \circledast$. Jun Tang ${ }^{3} \cdot$ Joshua Schrecker $^{4} \cdot$ Cheryl Hild $^{4}$
}

Published online: 8 October 2018

(c) The Author(s) 2018

\begin{abstract}
Background and Objective Aegis Sciences Corporation developed a test (InterACT Rx ${ }^{\mathrm{TM}}$ ) that objectively and definitively identifies substances known to interact with drug-drug interaction-prone medications commonly prescribed in the treatment of chronic pain and behavioral health disorders. The objective of this study was to assess the severity of identified drug-drug interactions, the reduction in the frequency and severity of identified drug-drug interactions, and the impact of the test on healthcare utilization.

Methods Patients with chronic pain, behavioral health disorders, or both who had one or more drug-drug interaction tests and one or more drug-drug interactions identified in the study period were included. Drug-drug interaction test results described the number and severity of interactions and detected substances involved in drug-drug interactions. Patients' electronic medical records were obtained to analyze outpatient visits and prescription medications. The cost of outpatient visits was based on the Medicare Physician Fee Schedule. Outcomes were compared between the pre- and post-study index periods to determine the impact of the drug-drug interaction test on patient care.

Results A total of 262 patients were included. The majority of drug-drug interactions detected (77.9\%) at index were of moderate severity. The number of monthly all-cause and pain-related outpatient visits was reduced in the post-index period compared with the pre-index period (0.74-0.54 and 0.69-0.49, respectively). Associated costs were reduced from US\$64.92 to US\$51.20, and from US\$62.42 to US\$47.62, ( $p<0.0001$ for both) for all-cause and pain-related outpatient visits, respectively. Follow-up drug-drug interaction testing for 43 patients revealed that previously reported drug-drug interactions at the index test were no longer identified in the subsequent test for $39.5 \%$ of patients.

Conclusions Employing a definitive test to detect substances whose interactions may cause adverse drug events can enhance a provider's insights, drive clinical decision making, and improve patient outcomes.
\end{abstract}

\section{Key Points}

At the time of this study, RJGA was Director, Navigant Consulting, Inc., New York, NY.

Electronic supplementary material The online version of this article (https://doi.org/10.1007/s40801-018-0143-z) contains supplementary material, which is available to authorized users.

Renée J. G. Arnold

renee.arnold@mssm.edu; rarnold@arnoldllc.com

1 Arnold Consultancy \& Technology, LLC, 15 West 72nd Street, 23rd Floor, New York, NY 10023, USA

2 Icahn School of Medicine at Mount Sinai, New York, NY, USA

3 Navigant Consulting, Inc., San Francisco, CA, USA

4 Aegis Sciences Corporation, Nashville, TN, USA
Traditional medication reconciliation processes are deficient in their ability to detect drug-drug interactions (DDIs).

The present study longitudinally evaluated the impact of a definitive DDI test on the frequency of DDIs identified in patients undergoing treatment for chronic pain and/or behavioral health conditions, patients' healthcare resource use, and providers' prescribing behavior.

There was a significant reduction in all-cause and painrelated outpatient visits and medication use between the pre- and post-index periods, including in the total number of prescriptions for opioid analgesics involved in DDIs. 


\section{Introduction}

For decades, it has been widely accepted that drug-drug interactions (DDIs) may cause significant adverse drug events (ADEs) [1]. According to the US Food and Drug Administration, interactions occur as a result of co-administration of multiple drugs or co-ingestion of over-thecounter products, herbal supplements, or food products and they may lead to variations in drug responses caused by a change in the systemic exposure of a medication [2]. By impacting drug response, DDIs predispose patients to an increased risk of ADEs resulting in emergency room visits, hospitalizations, and prolonged lengths of stay, thereby increasing costly healthcare resource use in addition to compromising patient safety [3-5]. While the impact of outpatient ADEs on healthcare utilization is well established, the national economic burden is difficult to calculate based on limited characterization of an associated cost in this setting. Although the per-incident cost for an inpatient ADE can exceed US\$3000, the economic impact of outpatient ADEs is also likely to be significant [6].

The Food and Drug Administration's Center for Drug Evaluation and Research estimated that 2 million serious ADEs occur in the USA each year, killing approximately 100,000 people [7]. Both prospective and retrospective analyses have demonstrated the mortality and cost associated with ADEs that cause hospitalization (prolonged length of stay and additional interventions) and those that occur in hospitals in the USA, the European Union, and South Africa [8-14]. Opioid-related DDIs, in particular, may have a major impact on healthcare costs in patients with chronic pain [15]. Studies on ADE rates and prevention have concluded that $35-40 \%$ of life-threatening or severe ADEs are preventable, and that $5-35 \%$ are caused by DDIs [16-19]. This suggests there is an opportunity to improve health outcomes by employing medication reconciliation processes that effectively prevent DDIs.

Traditional medication reconciliation processes are deficient in their ability to detect potential DDIs. The high rate of discrepancies between pharmacy claims data and provider medication lists offers an explanation as to why current methods to prevent DDIs are sometimes unsuccessful [20]. Furthermore, the use of non-prescription (over-the-counter) medications and supplements is rarely appropriately reported by patients. Hence, healthcare providers may fail to adequately identify all substances contributing to potential DDIs [21-23]. Enactment of legislative changes, including the introduction of the Medicare and Medicaid Electronic Health Records Incentive Program in 2011, have led to integrated clinical decision support (such as pop-up alerts to notify physicians of DDIs at the point of order entry) becoming increasingly available over time. Similarly, electronic health records have been available in a number of European hospitals for some time [24]. Unfortunately, the integration of automated clinical decision support into medication reconciliation practices is still limited in its ability to sufficiently prevent DDIs owing to the cumbersome nature of interruptive DDI alerts and reliance on appropriate self-report of medication use by patients. Research has shown that a significant number of DDI alerts are overridden by healthcare providers [25].

A solution to the gaps in care left by current medication reconciliation processes may be to improve the identification of concurrently ingested interacting substances via definitive DDI testing. Definitive identification of co-ingested substances that interact, combined with clinically actionable information pertaining to a reported interaction, may help reduce DDIs by allowing for more appropriate selection of medications and dosages. Aegis Sciences Corporation developed a test (InterACT $\mathrm{Rx}^{\mathrm{TM}}$ ) that objectively and definitively identifies substances known to interact with DDI-prone medications commonly prescribed in the treatment of chronic pain and behavioral health disorders [23, 26-29]. This test detects recently ingested substances, characterizes the severity associated with the interaction (moderate, severe, or contraindicated) and potential adverse outcomes, and improves healthcare providers' recognition of DDIs not discovered by traditional medication reconciliation practices. The present study was developed to longitudinally evaluate the impact of the test on the frequency of DDIs identified in patients undergoing treatment for chronic pain and/or behavioral health conditions, patients' healthcare resource use, and providers' prescribing behavior. Ultimately, it is our intent to encourage a greater use of DDI reporting to enhance other approaches (such as a dedicated clinical pharmacy unit in a trauma center [30]) used to reduce harmful ADEs.

\section{Methods}

\subsection{Study Design}

This institutional review board-approved (New England Institutional Review Board, 120170294) study was a retrospective, longitudinal, case-crossover, within-subject study of Aegis Sciences Corporation's DDI test. Certain groups of patients may be at an increased risk of ADEs, such as patients undergoing chronic pain management $[4,15,31]$. In recent years, the Centers for Medicare and Medicaid Services ran a campaign aimed at controlling pain, categorizing pain as the fifth vital sign. In response, there was an increase in the prescribing of opioid medications for pain management, often compromising patient safety and impacting health outcomes [32]. Thus, subjects in the current study included patients with chronic pain, 
behavioral health disorders, or both, who were being treated at either of two outpatient pain management facilities. Outcome measures included DDI test results, frequency of outpatient visits, and changes in prescribing behavior by prescribing providers. For patients with DDIs identified and reported, the results of any available subsequent DDI tests were assessed to determine whether the exact substances previously involved in a DDI were detected upon a follow-up test. Using electronic medical record (EMR) data, we analyzed changes in patients' prescription medications to determine the potential impact of DDI testing on healthcare providers' prescribing behavior.

The study period was from July 2015 to May 2018 (Fig. 1). Within this period, each patient's index date was defined as the date a DDI was first identified using the DDI test. The preindex date was defined as $\geq 90$ days preceding the index date; at least 180 days was considered for the post-index period.

\subsection{Study Population}

Sample size calculations were performed at $80 \%$ statistical power and a significance level of 0.05 . The study was powered to detect effects in the outcome variables between the preand post-index periods. Demographic characteristics of the patients in the study cohort did not show significant differences across the two treatment sites; hence, this was treated as a single study cohort group of 262 patients being treated at either of the two outpatient sites. Patients with chronic pain, behavioral health disorders, or both, receiving treatment from either site were eligible for inclusion. Patients were included if they were at least 18 years old at the index date, had at least one DDI test, had at least one DDI identified, had at least one pain diagnosis (as defined in Sect. 2.4), and had continuous healthcare coverage for $\geq 90$ days pre-index date through 180 days post-index date (based on EMR data). Patients with buprenorphine detected in a submitted specimen were excluded from the analysis, as this was a potential indicator that the patient was being treated for a condition that is outside the scope of this study.

\subsection{Data Retrieval for Included Patients}

Drug-drug interaction test results describing the number of DDIs identified per test, the severity of each reported DDI, and the exact substances detected that were involved in each DDI were retrieved from Aegis Sciences Corporation. Electronic medical record data on resource use (i.e., outpatient visits and prescribed medications) were retrieved for each site.
For patients included in this study, all DDI tests were ordered by healthcare providers at either of the two sites. The DDI test was completed by Aegis Sciences Corporation (Nashville, TN, USA); the test detects substances in urine specimens at or above a defined reporting threshold using definitive mass spectrometry methods. Clinically actionable information about all DDIs identified involving detected substances was reported to the ordering providers to inform patient care. Reports were delivered to the ordering providers electronically, and often in conjunction with medication compliance test results, on each respective patient's index date. Appendix 1 of the Electronic Supplementary Material (ESM) provides an excerpt from a laboratory report that includes DDI test results. Drug information included in reports was based on the contents of the Drug-Drug Interaction Module ${ }^{\mathrm{TM}}$ contained within FDB MedKnowledge $^{\mathrm{TM}}$, a comprehensive drug database utilized by healthcare providers to enhance clinical decision support (First Databank, Inc., South San Francisco, CA, USA).

The severity of each reported DDI was classified according to First Databank, Inc.'s definitions, described as follows in increasing order of severity (used with permission, CFirst Databank, Inc. All rights reserved):

- Moderate: "Interactions of moderate severity. The clinician should assess the patient's characteristics and act as needed. Actions required for moderate interactions include, but are not limited to, discontinuing one or both agents, adjusting dosage, altering administration scheduling, and providing additional patient monitoring."

- Severe: "Interactions that can produce serious consequences in most patients. Monitoring and/or titrating the agent(s) involved in severe interactions can significantly minimize the risk of adverse effects."

- Contraindicated: "Interactions involving drug combinations that generally should not be dispensed or administered to the same patient. These interactions include those indicated by a manufacturer label warning that indicates the contraindication regardless of clinical evidence or lack of clinical evidence to support the contraindication."

Electronic medical record data described the following demographic information for each patient: age, sex, and body mass index. In addition to providing demographic information, EMR data were used to quantify the number of office visits for each patient during the study period, the diagnosis codes associated with those visits, and prescription medication information for each patient (including medication type and dose).

Fig. 1 Study timeline

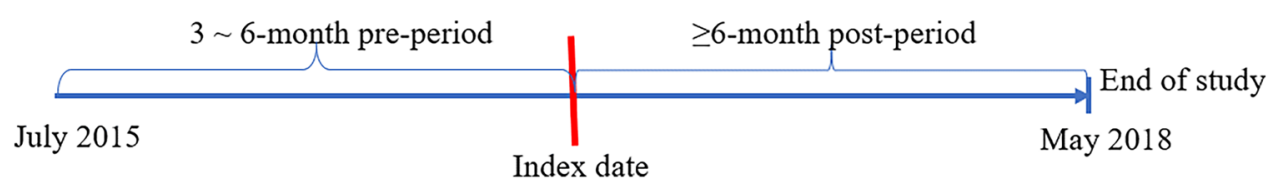




\subsection{Data Analysis}

The data, including DDI test results as well as the EMR utilization and prescribing information for each patient included in the study, were analyzed both for the control period (preindex) and the post-index period. Results were compared between the two periods to determine the potential impact of DDI testing on the frequency and severity of DDIs, patients' healthcare utilization and associated costs, and on healthcare providers' prescribing behavior. Calculations were performed at the individual patient level to evaluate pre- and post-index differences in the study outcome measures.

Patients' demographic information and DDI test results were reported, with age group and body mass index category being reported as nominal variables. Both the total number and cost of monthly all-cause outpatient visits were reported, as well as the number and cost of monthly outpatient visits identified as being specifically attributable to pain. Painrelated diagnoses were identified using the following codes from the ninth and tenth revisions of the International Classification of Diseases, Clinical Modification (ICD-CM) systems:

1. ICD-9-CM: 338.2 or 338.4 ; or

2. ICD-10-CM: Z79.891, Z79.899, F11.20, M54.5, G89.4, G89.29, Z51.81, M54.16, M54.2, M96.1, M51.36, M54.17, M41.9, M47.817, M54.12, M47.816, M79.7, F10.20, Z03.89, M51.26, M79.1, F19.20, M46.1, M48.06, or F41.1.

Payer-borne costs of office visits were applied to estimate the financial burden of chronic disease state management. The cost of each visit was the non-facility provider payment described in the Centers for Medicare and Medicaid Services' 2017 Medicare Physician Fee Schedule. Exact procedure codes were based on EMR data. Pain-related procedure (CPT) codes were examined manually to ascertain that they were actually related to pain (see Appendix 2 of the ESM). Overall healthcare costs were calculated based on the allcause procedure codes and pain-related costs were calculated based on the pain-related procedure codes.

Drug-drug interaction test results from the pre- and postindex periods were compared to assess how many patients showed a change in substance use for those specific substances involved in the identified DDIs. For patients with prescriptions for medications involved in DDIs identified in the pre-index period, EMR data were used to recognize changes in medication therapy and to determine whether the healthcare provider prescribed the same medication and at what dose in the post-index period. Drug classes and severity levels of DDIs identified at each point were also reported. For patients with limited information in the EMR data on prescription medication changes, but who had a second DDI test completed, DDI resolution was recorded if at least one of the involved substances was not detected in the DDI test immediately following the index date test.

\subsection{Statistical Methods}

This study assessed changes in patient outcomes data over time using comparative statistics for a single paired sample. Paired $t$ tests or Wilcoxon signed rank tests were used for continuous variables. Chi-squared tests were used for nominal variables. All tests were completed using SAS Version 9.4 .

\section{Results}

A total of 262 patients receiving care from 13 different practitioners at the two facilities were included (Table 1). At index, the mean age of included patients was 56.70 years (standard deviation of 11.24 years), and mean body mass index was $30.80 \mathrm{~kg} / \mathrm{m}^{2}$ (standard deviation of $7.84 \mathrm{~kg} / \mathrm{m}^{2}$ ). The average number of DDIs identified at index was 1.82 (standard deviation of 1.32) per patient, most of which were moderate in nature.

Patients experienced significantly more all-cause outpatient visits per month in the pre-index period compared with the post-index period (Table 2, Fig. 2). This translated to significantly greater all-cause costs associated with outpatient visits in the pre-index period compared with the post-index period (US\$64.92 pre-index vs. US $\$ 51.20$ postindex; $p<0.0001)$. A similar decrease in healthcare utilization costs for pain-related visits only was observed in the post-index period compared with the pre-index period. The per-patient monthly cost of pain-related outpatient visits decreased by $23.7 \%$ (US $\$ 62.42$ vs. US $\$ 47.63 ; p<0.0001$ ) from the pre-index to post-index periods.

Evaluations of change in the frequency of interactions and provider prescribing patterns are summarized in Table 3. Electronic medical record data revealed that for most patients (191) at least one prescription in the postindex period for a medication was previously involved in a DDI. Between the pre- and post-index periods, 150 of these patients saw a change in the drug classes detected by the DDI test, indicating a change in the healthcare provider's prescribing behaviors following index DDI test results. Additionally, the total number of prescriptions for opioid analgesics involved in DDIs identified in all patients within the cohort decreased by $12.7 \%$ in the post-index period (1418) compared with the pre-index period (1625). Further assessments were completed for 43 patients with follow-up DDI test results available subsequent to the index DDI test. Within this group of patients, $39.5 \%$ no longer had DDIs identified that were previously reported on the index date 
Table 1 Patient characteristics at index

\begin{tabular}{lc}
\hline Variable & $N(\%)$ \\
\hline All patients & $262(100)$ \\
Age group, years & \\
$18-44$ & $37(14.12)$ \\
$45-54$ & $69(26.34)$ \\
$55-64$ & $89(33.97)$ \\
$65+$ & $67(25.57)$ \\
Sex & \\
Female & $173(66.03)$ \\
Male & $89(33.97)$ \\
BMI group, kg/m ${ }^{2}$ & $7(2.67)$ \\
Underweight, $<18.5$ & $55(20.99)$ \\
Normal weight, 18.5-24.9 & $62(23.66)$ \\
Overweight, 25-29.9 & $130(49.62)$ \\
Obese, $\geq 30.0$ & $8(3.05)$ \\
Unknown & \\
DDI severity at index & \\
Contraindicated & $46(17.56)$ \\
Severe & $73(27.86)$ \\
Moderate & $204(77.86)$ \\
\hline
\end{tabular}

$B M I$ body mass index, $D D I$ drug-drug interaction

${ }^{\text {a }}$ Percentages not mutually exclusive

DDI test, including substantial reductions in contraindicated and severe interactions.

\section{Discussion}

This study indicates that the DDI test is an effective drug safety intervention in reducing the frequency of identified DDIs, healthcare utilization, and costs among the non-addiction chronic pain and behavioral health patient populations. Attention to ADEs caused by DDIs has been mostly focused on the inpatient hospital setting or emergency department $[3,4,33]$. However, most medication is prescribed and used outside of hospitals and is managed by patients or caregivers in homes or workplaces. This study demonstrates the effects of the DDI test on outpatient health resource utilization, overcoming some of the barriers associated with traditional medication reconciliation processes, indicating that the DDI test can contribute to substantial healthcare savings in this population.

In this study, $77.9 \%$ of the patients had moderate DDIs identified, and $45.4 \%$ had either a contraindicated and/ or severe DDI identified through the definitive DDI test. These reported DDIs require a prompt patient-centered intervention so that patients can receive appropriate instructions from clinicians to prevent ADEs. Our results also showed that changes were made in patients' prescription regimens by their healthcare providers in the majority of patients with a reported DDI. In turn, patients had reduced healthcare utilization and costs.

The prevalence of opioid therapy for pain management demonstrates the growing clinical need to objectively identify potential DDIs to optimize prescription
Table 2 Healthcare resource utilization comparison

\begin{tabular}{|c|c|c|c|}
\hline Monthly outpatient visits per patient & Pre-index period & Post-index period & $P$ value \\
\hline All-cause visits ${ }^{\mathrm{a}}$ & & & $<0.0001$ \\
\hline Mean (SD) & $0.74(0.23)$ & $0.54(0.30)$ & \\
\hline Median & 0.70 & 0.40 & \\
\hline Range & $0.30-2.00$ & $0.20-2.00$ & \\
\hline Cost (2017) of all-cause visits ${ }^{b}$ & & & $<0.0001$ \\
\hline Mean (SD) & US\$64.92 (US\$24.52) & US\$51.20 (US\$27.60) & \\
\hline Median & US\$59.86 & US\$41.04 & \\
\hline Range & US\$17.89-US\$154.01 & US\$16.31-US\$186.41 & \\
\hline Pain-related visits ${ }^{\mathrm{a}}$ & & & $<0.0001$ \\
\hline Mean (SD) & $0.69(0.28)$ & $0.49(0.29)$ & \\
\hline Median & 0.70 & 0.40 & \\
\hline Range & $0.20-2.00$ & $0.20-2.00$ & \\
\hline Cost of pain-related visits ${ }^{b}$ & & & $<0.0001$ \\
\hline Mean (SD) & US\$62.42 (US\$30.42) & US\$47.63 (US\$27.72) & \\
\hline Median & US\$56.13 & US\$38.38 & \\
\hline Range & US\$3.46-US\$154.01 & US\$7.24-US\$152.23 & \\
\hline
\end{tabular}


Fig. 2 Mean outpatient visits

Outpatient Visits, mean per patient

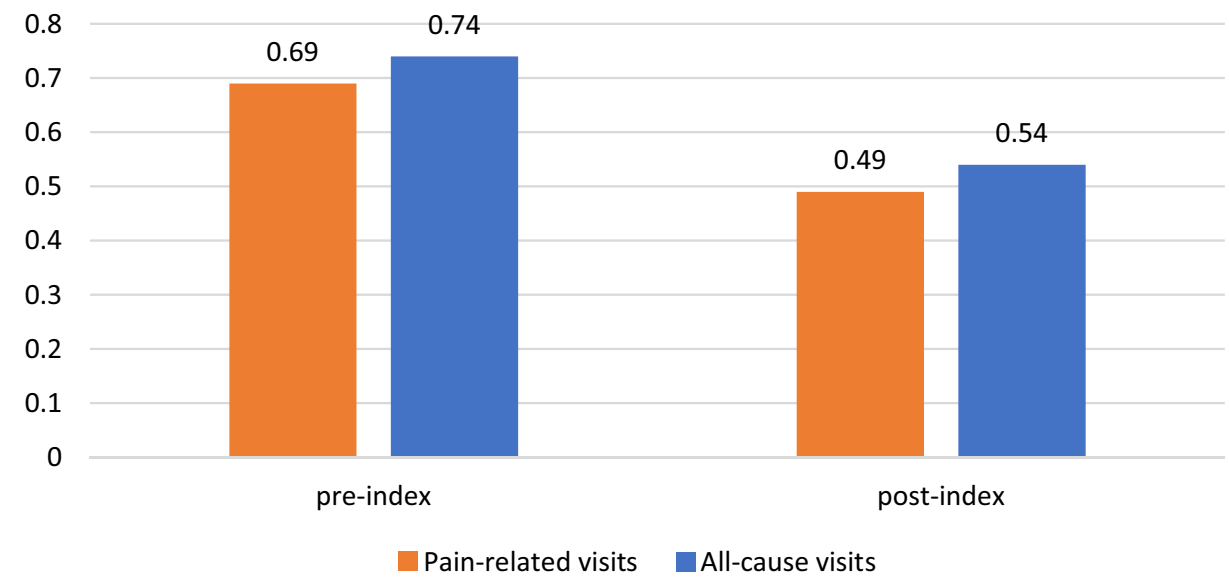

Table 3 Drug-drug interaction (DDI)-related medication prescription status

\begin{tabular}{lll}
\hline Post-index period medication prescription status & $N(\%)$ & \\
\hline Patients with medication prescriptions & $191(100)$ & \\
No change compared to pre-index period & $55(28.8)$ & \\
Altered regimen compared to pre-index period & $15(7.9)$ & \\
Dose change compared to pre-index period & $11(5.8)$ & \\
Reduced dose & $6(54.5)$ & \\
Increased dose & $5(45.5)$ & Post-index period \\
Drug class change compared to pre-index period & $150(78.5)$ & $4(6.7)$ \\
Patients without medication prescriptions & $71(100)$ & $9(15.0)$ \\
Patients who had another DDI test in the post-index period & $43(60.6)$ & $32(53.3)$ \\
DDI severity & Pre-index period & $11(18.3)$ \\
Contraindicated & $16(26.7)$ & $15(25.0)$ \\
Severe & $33(55.0)$ & \\
Moderate & $0(0.0)$ & $17(39.53)$ \\
None & & \\
Patients whose DDIs were not present post-index &
\end{tabular}

therapy. The unmet need is supported by our study results, which showed that one of the substances most commonly involved in DDI-related prescriptions was opioids, and opioid use was reduced by $12.7 \%$ after utilization of the DDI test. There is a growing understanding that opioids, while sometimes necessary to manage chronic pain, may put patients at an increased risk for ADEs, even when utilized appropriately [34]. Employing objective drug safety interventions can improve a provider's understanding of medications that are increasing their patients' risk of opioid-related ADEs, lending additional, objective clinical decision support during the prescribing process. Endowing providers with DDI test results can also be impactful in offsetting current limitations of medication reconciliation (such as a lack of effective communication between prescribing physicians during transitions of care).
While most studies of the impact of DDIs have used claims data, the current study used EMR data. There are several strengths of EMR data [4, 35]. First, extensive and upto-date longitudinal EMR data have minimal observer bias. Second, real-world patient utilization data reflect the actual effects of Aegis Sciences Corporation's DDI testing program on patient management by the provider. This helps healthcare providers decide which members of a given population to target with a particular medication. There are also challenges in the use of EMR data. In our study, we encountered incomplete medication prescription records, different coding systems among different sites and providers, lack of administrative data for the medication costs, and availability of only outpatient visit information. No information was available on emergency department or inpatient treatments for the majority of the patients in the study cohort. In addition, 
several procedures were recorded that did not have associated costs. To reflect the real cost to the healthcare providers, these costs were not included in the analysis. Thus, the reported healthcare utilization costs from this study may be underestimated. However, those cases occurred in both preand post-index periods, thus limiting potential bias in the present study.

In this study, patients were assumed to be at the greatest risk of ADEs when DDI results showed the highest number of DDIs identified, irrespective of severity level. Exact risk, however, is likely dependent upon both the number and severity of DDIs. For example, it may be preferable for a patient to face the risk of ADEs associated with one severe DDI as opposed to three moderate DDIs. Because exact risk is difficult to calculate precisely and is highly patient-specific, the simplifying assumption of DDI change was made that the DDIs detected at the index were resolved in the follow-up DDI test. That is, if a patient had the same DDI identified at the follow-up test, the patient was not considered to have a resolved DDI; otherwise, if the exact substances involved in the index interactions were not shown in the later test, the patient was considered to have a resolved DDI.

Last, as this is an observational study, one can only identify an association, rather than a causal relationship, between the DDI test and a reduction in the frequency of identified DDIs, healthcare utilization, and costs among the non-addiction chronic pain and behavioral health patient populations. In addition, the research relied on retrospective data and it was not possible to determine if the change in DDIs actually resulted in fewer ADEs. While the present data suggest fewer ADEs are occurring (as fewer severe/contraindicated interactions were detected), a controlled longitudinal prospective study is needed to accurately assess this outcome. This study type may also allow for better incorporation of additional cost data associated with other patient- and utilization-related outcomes.

\section{Conclusion}

In this observational study, the post-index period was associated with a significant reduction in all-cause outpatient visits and all-cause and pain-related costs per month compared with the pre-index period. Additionally, the total number of prescriptions for opioid analgesics involved in DDIs identified in all patients within the cohort decreased by $12.7 \%$ in the post-index period, with substantial reductions in contraindicated and severe interactions. The study showed that employing a definitive test to detect substances whose interactions may cause ADEs can enhance a provider's insights, drive clinical decision making, and improve patient outcomes.

\section{Compliance with Ethical Standards}

Funding Funding for this research was provided by Aegis Sciences Corporation to Navigant Consulting, Inc.

Conflict of interest Joshua Schrecker and Cheryl Hild are employees of Aegis Sciences Corporation. Renée J. G. Arnold was an employee of Navigant Consulting, Inc. at the time of this study. Jun Tang is an employee of Navigant Consulting, Inc.

Open Access This article is distributed under the terms of the Creative Commons Attribution-NonCommercial 4.0 International License (http://creativecommons.org/licenses/by-nc/4.0/), which permits any noncommercial use, distribution, and reproduction in any medium, provided you give appropriate credit to the original author(s) and the source, provide a link to the Creative Commons license, and indicate if changes were made.

\section{References}

1. Huang SM, Strong JM, Zhang L, Reynolds KS, Nallani S, Temple $R$, et al. New era in drug interaction evaluation: US Food and Drug Administration update on CYP enzymes, transporters, and the guidance process. J Clin Pharmacol. 2008;48(6):662-70. https ://doi.org/10.1177/0091270007312153.

2. US Food and Drug Administration. Drug development and drug interactions. 2018. https://www.fda.gov/drugs/developmentappr ovalprocess/developmentresources/druginteractionslabeling/ ucm080499.htm\#overview. Accessed 24 Sep 2018.

3. Budnitz DS, Pollock DA, Weidenbach KN, Mendelsohn AB, Schroeder TJ, Annest JL. National surveillance of emergency department visits for outpatient adverse drug events. JAMA. 2006;296(15):1858-66. https://doi.org/10.1001/ jama.296.15.1858.

4. Taylor R Jr, V Pergolizzi J Jr, Puenpatom RA, Summers KH. Economic implications of potential drug-drug interactions in chronic pain patients. Expert Rev Pharmacoecon Outcomes Res. 2013;13(6):725-34. https://doi.org/10.1586/14737 167.2013.851006.

5. Moura CS, Acurcio FA, Belo NO. Drug-drug interactions associated with length of stay and cost of hospitalization. J Pharm Pharm Sci. 2009;12(3):266-72.

6. Hug BL, Keohane C, Seger DL, Yoon C, Bates DW. The costs of adverse drug events in community hospitals. Jt Comm J Qual Patient Saf. 2012;38(3):120-6.

7. US Food and Drug Administration. Preventable adverse drug reactions: a focus on drug interactions. 2018. https://www.fda. gov/Drugs/Developmentapprovalprocess/Developmentresources/ Druginteractionslabeling/ucm110632.htm. Accessed 24 Sep 2018.

8. Bouvy JC, De Bruin ML, Koopmanschap MA. Epidemiology of adverse drug reactions in Europe: a review of recent observational studies. Drug Saf. 2015;38(5):437-53. https://doi.org/10.1007/ s40264-015-0281-0.

9. Brvar M, Fokter N, Bunc M, Mozina M. The frequency of adverse drug reaction related admissions according to method of detection, admission urgency and medical department specialty. BMC Clin Pharmacol. 2009;9:8. https://doi.org/10.1186/1472-6904-9-8. 
10. Jemal A, Ward E, Hao Y, Thun M. Trends in the leading causes of death in the United States, 1970-2002. JAMA. 2005;294(10):1255-9. https://doi.org/10.1001/jama.294.10.1255.

11. Mouton JP, Mehta U, Parrish AG, Wilson DP, Stewart A, Njuguna $\mathrm{CW}$, et al. Mortality from adverse drug reactions in adult medical inpatients at four hospitals in South Africa: a cross-sectional survey. Br J Clin Pharmacol. 2015;80(4):818-26. https://doi. org/10.1111/bcp.12567.

12. Veeren J, Weiss M. Trends in emergency hospital admissions in England due to adverse drug reactions: 2008-2015. J Pharm Health Serv Res. 2017;8:5-11.

13. Formica D, Sultana J, Cutroneo PM, Lucchesi S, Angelica R, Crisafulli $\mathrm{S}$, et al. The economic burden of preventable adverse drug reactions: a systematic review of observational studies. Expert Opin Drug Saf. 2018;17(7):681-95. https://doi. org/10.1080/14740338.2018.1491547.

14. Sultana J, Cutroneo P, Trifiro G. Clinical and economic burden of adverse drug reactions. J Pharmacol Pharmacother. 2013;4(Suppl. 1):S73-7. https://doi.org/10.4103/0976-500X.120957.

15. Pergolizzi JV Jr, Ma L, Foster DR, Overholser BR, Sowinski KM, Taylor R Jr, et al. The prevalence of opioid-related major potential drug-drug interactions and their impact on health care costs in chronic pain patients. J Manag Care Spec Pharm. 2014;20(5):46776. https://doi.org/10.18553/jmcp.2014.20.5.467.

16. Adverse drug reactions and drug-drug interactions: consequences and costs. American Forensic Medical Specialists. 2018. https ://www.amfs.com/news/articles-from-our-experts/adverse-drugreactions-and-drug-drug-interactions-consequences-and-costs/. Accessed 27 Jun 2018.

17. Bates DW, Cullen DJ, Laird N, Petersen LA, Small SD, Servi $\mathrm{D}$, et al. Incidence of adverse drug events and potential adverse drug events: implications for prevention. ADE Prev Study Group. JAMA. 1995;274(1):29-34.

18. Wright A, Feblowitz J, Phansalkar S, Liu J, Wilcox A, Keohane C, et al. Preventability of adverse drug events involving multiple drugs using publicly available clinical decision support tools. Am J Health Syst Pharm. 2012;69(3):221-7. https://doi.org/10.2146/ ajhp110084.

19. Magro L, Moretti U, Leone R. Epidemiology and characteristics of adverse drug reactions caused by drug-drug interactions. Expert Opin Drug Saf. 2012;11(1):83-94. https://doi.org/10.1517/14740 338.2012.631910.

20. Comer D, Couto J, Aguiar R, Wu P, Elliott DJ. Usefulness of pharmacy claims for medication reconciliation in primary care. Am J Manag Care. 2015;21(7):486-93.

21. Gleason KM, McDaniel MR, Feinglass J, Baker DW, Lindquist L, Liss D, et al. Results of the Medications at Transitions and Clinical Handoffs (MATCH) study: an analysis of medication reconciliation errors and risk factors at hospital admission. J Gen Intern Med. 2010;25(5):441-7. https://doi.org/10.1007/s1160 6-010-1256-6.

22. Qato DM, Alexander GC, Conti RM, Johnson M, Schumm P, Lindau ST. Use of prescription and over-the-counter medications and dietary supplements among older adults in the United States. JAMA. 2008;300(24):2867-78. https://doi.org/10.1001/ jama.2008.892.

23. Qato DM, Wilder J, Schumm LP, Gillet V, Alexander GC. Changes in prescription and over-the-counter medication and dietary supplement use among older adults in the United States, 2005 vs 2011. JAMA Intern Med. 2016;176(4):473-82. https:// doi.org/10.1001/jamainternmed.2015.8581.

24. Cars T, Wettermark B, Malmstrom RE, Ekeving G, Vikstrom B, Bergman U, et al. Extraction of electronic health record data in a hospital setting: comparison of automatic and semi-automatic methods using anti-TNF therapy as model. Basic Clin Pharmacol Toxicol. 2013;112(6):392-400. https://doi.org/10.1111/ bcpt. 12055 .

25. Nanji KC, Slight SP, Seger DL, Cho I, Fiskio JM, Redden LM, et al. Overrides of medication-related clinical decision support alerts in outpatients. J Am Med Inform Assoc. 2014;21(3):48791. https://doi.org/10.1136/amiajnl-2013-001813.

26. Overholser BR, Foster DR. Opioid pharmacokinetic drug-drug interactions. Am J Manag Care. 2011;17(Suppl. 11):S276-87.

27. Kennedy WK, Jann MW, Kutscher EC. Clinically significant drug interactions with atypical antipsychotics. CNS Drugs. 2013;27(12):1021-48. https://doi.org/10.1007/s4026 3-013-0114-6.

28. Spina E, Trifiro G, Caraci F. Clinically significant drug interactions with newer antidepressants. CNS Drugs. 2012;26(1):39-67. https://doi.org/10.2165/11594710-000000000-00000.

29. Moody DE. Metabolic and toxicological considerations of the opioid replacement therapy and analgesic drugs: methadone and buprenorphine. Expert Opin Drug Metab Toxicol. 2013;9(6):67597. https://doi.org/10.1517/17425255.2013.783567.

30. Hamblin S, Rumbaugh K, Miller R. Prevention of adverse drug events and cost savings associated with PharmD interventions in an academic Level I trauma center: an evidence-based approach. J Trauma Acute Care Surg. 2012;73(6):1484-90. https://doi. org/10.1097/TA.0b013e318267cd80.

31. Pergolizzi JV Jr, Labhsetwar SA, Amy Puenpatom R, Ben-Joseph R, Ohsfeldt R, Summers KH. Economic impact of potential CYP450 pharmacokinetic drug-drug interactions among chronic low back pain patients taking opioids. Pain Pract. 2012;12(1):4556. https://doi.org/10.1111/j.1533-2500.2011.00503.x.

32. Tompkins DA, Hobelmann JG, Compton P. Providing chronic pain management in the "Fifth Vital Sign" era: historical and treatment perspectives on a modern-day medical dilemma. Drug Alcohol Depend. 2017;173(Suppl. 1):S11-21. https://doi.org/10.1016/j. drugalcdep.2016.12.002.

33. Dechanont S, Maphanta S, Butthum B, Kongkaew C. Hospital admissions/visits associated with drug-drug interactions: a systematic review and meta-analysis. Pharmacoepidemiol Drug Saf. 2014;23(5):489-97. https://doi.org/10.1002/pds.3592.

34. Shehab N, Lovegrove MC, Geller AI, Rose KO, Weidle NJ, Budnitz DS. US emergency department visits for outpatient adverse drug events, 2013-2014. JAMA. 2016;316(20):2115-25. https:// doi.org/10.1001/jama.2016.16201.

35. Ernst FR, Mills JR, Berner T, House J, Herndon C. Opioid medication practices observed in chronic pain patients presenting for all-causes to emergency departments: prevalence and impact on health care outcomes. J Manag Care Spec Pharm. 2015;21(10):925-36. https://doi.org/10.18553/ jmcp.2015.21.10.925. 